\title{
A Study on Incidence of Malignancy in Multinodular Goitre
}

\author{
Dr.S.Vijayakumar M.S. ${ }^{1}$, Dr.R.Alagar samy M.S. ${ }^{2}$, \\ ${ }^{1}$ Chief civil surgeon, ${ }^{2}$ Senior Resident, Department of General surgery, Government Medical college and \\ ESIC Hospital, Coimbatore, Tamilnadu, India.
}

\begin{abstract}
Introduction: Multi-nodular goitre $(M N G)$ is one of the common presentations of various thyroid diseases. Thyroid nodules have been reported to be found in $4 \%$ to $7 \%$ of the population on neck palpation. Although MNG was traditionally thought to be at a low risk for malignancy as compared to its single-nodule counterpart, various studies have reported a significant risk. The objective of this study was to determine the incidence and the type of thyroid carcinoma (TC) in multi-nodular goitre by doing the Histopathological examination of thyroidectomy specimens. Materials and Methods: This prospective, observational study was carried out in the Department of Surgery at Government medical college and ESIC Hospital, coimbatore, India from Jan 2015 to Dec 2015.All the patients with multi-nodular goitre with or without thyrotoxicosis were evaluated and they were offered surgery as the treatment for suspicious findings, compressive symptoms and thyrotoxicosis. The specimens were subjected to a Histopathological evaluation to determine the incidence and the types of various malignancies in MNG. Results: Among the 29 MNG cases which were studied, 3 (10.34\%) cases contained malignant foci. Among them, papillary carcinoma 2(33.33\%) was the most common type of malignancy which was observed. Conclusion: The incidence of malignancy in MNG is quite significant and it is not very low as was thought before. Due to the risk of occult malignancy, all the patients with multi-nodular goitres who are treated conservatively need a close follow up for malignancy.
\end{abstract}

Keywords: Thyroidectomy, Thyrotoxicosis , Multinodular Goitre , Histopathology

\section{Introduction}

MNG is one of the common presentations of various thyroid diseases. Thyroid nodules have been reported to be found in $4 \%$ to $7 \%$ of the population on neck palpation and in $30 \%$ to $50 \%$ of the population by ultrasonography (USG) ${ }^{1,2,3}$. A long standing and hitherto unresolved issue is whether MNG is significantly associated with malignancy ${ }^{4}$. MNG had been traditionally thought to be at a low risk for malignancy as compared to a solitary nodule thyroid ${ }^{5,6,7}$. However, various studies have reported a 7 to $17 \%$ incidence of malignancy in $\mathrm{MNG}^{6,8,9}$. The most common variety of malignancy which has been documented in the literature is papillary carcinoma ${ }^{10}$. The management of a solitary nodule has been refined by FNAC, unlike MNG, in which a nodule of a carcinoma can't be differentiated clinically or sonologicaly amidst other benign nodules ${ }^{4,10}$.The incidence of the thyroid malignancy ranges from $0.9 \%$ to $13 \%$ in different parts of world ${ }^{11}$. Such an incidence increases further if cases of occult carcinoma are also taken into consideration. The exposure to ionizing radiation and the availability of more sensitive diagnostic tests may be the possible explanations for a worldwide increase in the incidence of thyroid carcinoma ${ }^{12}$.The objective of this study was to determine the incidence and the types of various thyroid malignancies in multi-nodular goitre by doing the histopathological examination of total thyroidectomy specimens.

\section{Materials And Methods}

This prospective, observational study was carried out in the Department of Surgery at the Government medical college and ESIC Hospital, coimbatore, India from Jan 2015 to Dec 2015. All the patients with goitre were examined clinically and sonologically. The patients with multi-nodular goitre with or without thyrotoxicosis and those who subsequently underwent total thyroidectomy were selected for the study. Patients with a solitary nodule, Grave's disease, recurrent goitre, a proven thyroid malignancy and metastatic cervical lymphadenopathy with occult primary were excluded from the study. The patients in whom the nodules were not detected clinically and/or sonologically, but were revealed during the operation or on histopathological evaluation were also excluded from the study. During the pre-operative evaluation, all the selected patients underwent a detailed clinical examination, biochemical investigations including the thyroid hormone assay and the evaluation of the serum calcium levels, thyroid ultrasonography and indirect laryngoscopy to check the vocal cord movements. FNAC was performed only in cases with a suspicious nodule (rapidly growing, hard, irregular nodules, the presence of microcalcification, etc) which was detected during the clinical examination and on USG. The patients were offered surgery as a treatment based on the suspicious findings during the diagnostic work-up, equivocal results from the various investigations, the compressive symptoms, thyrotoxicosis and cosmesis. In all the selected cases of MNG, total thyroidectomy was performed following the 
identification and the preservation of the recurrent laryngeal nerves and the parathyroid glands. After the surgery, all the thyroid specimens were sent for a histopathological evaluation. All pre-operative, operative and the post-operative findings were recorded in detail in a standard format and the results were evaluated.

\section{Results}

In this prospective study, we had 29 cases of goitre. Of the $29 \mathrm{MNG}$ cases, $26(89.66 \%)$ were females and $3(10.34 \%)$ were males, with a striking female predominance(Table1:2). A majority of the patients were in the second to fourth decades of life (Table1:1). The histopathology of the specimens revealed that 3 patients had a malignant focus and so the incidence of $\mathrm{Ca}$ in MNG was 10\% in our study (Table1:3) Among the malignancies, papillary carcinoma (66.66\%) was the commonest type which was observed in MNG in our study

Table1:1 Age wise distribution of MNG

\begin{tabular}{|l|l|l|l|}
\hline Sl.no & Age group & No of patients & $\%$ of patients \\
\hline 1. & Less than 20 & 0 & 0 \\
\hline 2. & $20-40$ & 15 & 51.72 \\
\hline 3. & $40-60$ & 13 & 44.82 \\
\hline 4. & Above 60 & 1 & 3.44 \\
\hline
\end{tabular}

Table1:2 Sex wise distribution of MNG

\begin{tabular}{|l|l|l|l|}
\hline Sl.no & Sex & No of patients & $\%$ of patients \\
\hline 1. & Male & 3 & 10.34 \\
\hline 2. & Female & 26 & 89.66 \\
\hline
\end{tabular}

Table1:3 Type of malignancy in MNG

\begin{tabular}{|l|l|l|l|}
\hline Sl. No & Type of carcinoma & No of patients & $\%$ of patients \\
\hline 1. & Papillary carcinoma of thyroid & 2 & 6.89 \\
\hline 2. & Follicular carcinoma of thyroid & 1 & 3.44 \\
\hline 3. & Medullary carcinoma of thyroid & 0 & 0 \\
\hline 4. & Anaplastic carcinoma of thyroid & 0 & 0 \\
\hline 5. & Beningn & 26 & 89.66 \\
\hline
\end{tabular}

Table1:4 Sex wise distribution of Malignancy

\begin{tabular}{|l|l|l|l|}
\hline Sl.no & Sex & No of patients & $\%$ of patients \\
\hline 1. & Male & 3 & 100 \\
\hline 2. & Female & 0 & 0 \\
\hline
\end{tabular}

\section{Discussion}

Multi-nodular goitre (MNG) is defined as the palpation of multiple distinct nodules in the enlarged thyroid gland. The aetiopathogenesis of MNG is not very clear. A mild dietary deficiency of iodine, slight impairment of hormone synthesis, increased iodide clearance from the kidneys and the presence of thyroid stimulating immunoglobulins have been suggested as the various causes. ${ }^{4}$.Thyroid nodules have been reported in $4 \%$ to $7 \%$ of the population on neck palpation (the incidence increases with age) and in $30 \%$ to $50 \%$ of the population by ultrasonography ${ }^{1,2,3}$. It has been observed that $50.5 \%$ of the solitary nodules which are felt on palpation are actually a part of the multi-nodular goitre . The appreciation of the nodules may be hampered by the presence of a short and thick neck. MNG had been traditionally thought to be at a low risk for malignancy as compared to a solitary nodule thyroid ${ }^{5,6,7}$. However, various studies have shown that the risk is quite high in MNG also. A study which was conducted by Benzarti et al in Tunis found a $9.5 \%$ incidence of malignancy in MNG ${ }^{11}$, whereas Sarajevo reported an $8 \%$ incidence of malignancy in MNG in his study ${ }^{11}$. Prades et al from France, however, reported quite a high incidence ie $12.2 \%{ }^{11}$. The most common variety of malignancy which has been documented in the literature is papillary carcinoma ${ }^{7,10}$. The incidence of carcinoma in MNG in our study was $10 \%$ and the most common type of malignancy which was observed was papillary carcinoma $(66.66 \%)$. This was consistent with the observations which were made by Benzarti et al in Tunis ${ }^{11}$.A thyroid nodule should be viewed with suspicion if it is seen as a dominant nodule in the MNG, which is hard, irregular, fixed, and rapidly increasing, which is seen along with cervical lympadenopathy, recurrent laryngeal nerve palsy, extremes of age and the male sex. A patient with a history of neck irradiation or a family history of thyroid carcinoma (TC) should make the suspicion of MNG strong ${ }^{11}$.High-frequency, real-time ultrasonography and fine-needle aspiration cytology (FNAC) are the indispensible tools which are used in the pre-operative evaluation of MNG for malignant foci. The important sonographic findings which are suggestive of malignancy in the thyroid nodules are micro-calcifications (which are present in about $22 \%$ of the thyroid cancers), irregular margins of the nodules a complex echogenicity and smaller nodules ${ }^{1}$. It has been postulated that the thyroid cancers would have manifested with more overt signs and symptoms of local invasion or metastasis by the time they had reached a significant size ${ }^{1}$.FNAC is a fast and inexpensive investigation which can be done to obtain 
cellular samples ${ }^{1}$. A series of reviews have reaffirmed its importance in the assessment of the thyroid nodules. However, a negative FNAC report does not exclude with certainty the possibility of a carcinoma, especially in MNG, where the error in sampling the right area is greater ${ }^{6,7}$. FNAC of a suspicious nodule under USG guidance is of great help. Thyroid carcinomas account for $1 \%$ of all the malignancies and they are the most common endocrine tumours ${ }^{11}$. The incidence of TC varies considerably in different regions of the world. Globally, the incidence of TC has increased by up to five-fold during the past 60 years ${ }^{5}$. The tumours are rare in children and their frequency increases with age. Overall, females have a higher incidence of TC ${ }^{11}$. Ionizing radiation, iodine deficiency and other factors have been attributed for the increase in TC, but these findings are inconsistent. Hormonal factors, lactation suppressive drugs and fertility medications have been implicated for the high incidence of TC in females. However, recent studies have reported no significant risk which has been associated with the use of hormone replacement therapy or fertility drugs ${ }^{11}$.It also has been proposed that the availability of better and more sensitive diagnostic tools may be responsible for the increasing incidence of TC ${ }^{12}$.Papillary micro-carcinoma is one subtype of papillary carcinoma, which was a frequent incidental finding in many autopsy studies ${ }^{12}$. The World Health Organization has defined it as a papillary thyroid carcinoma which measures $\leq 10 \mathrm{~mm}$ in the greatest dimension ${ }^{12}$.

\section{Conclusion}

We conclude that the risk of malignancy in multi-nodular goitre is not as low as it was thought before and that it is quite significant. Due to the risk of occult malignancy, all the patients with multinodular goitre who have been treated conservatively need a close follow up for malignancy.

\section{Source of Support: Nil \\ Conflict of Interest: Nil.}

\section{References}

[1] Pang H-N, Chen C-M. The incidence of cancer in nodular goitres. Ann Acad Med Singapore 2007; 36:241-43.

[2] Belfiore A, La Rosa GL, Padova G, Sava L, Ippolito O, Vigneri R. The frequency of cold thyroid nodules and thyroid malignancies in patients from an iodine-deficient area. Cancer 1987; 60:3096-102

[3] Mazzaferri EL, de los Santos ET, Rofagha-Keyhani S. Solitary thyroid nodule: diagnosis and management. Med Clin North Am 1988; 72: 1177-211.

[4] Abu-Eshy SA, Khan AR, Khan GM, Al-Humaidi MA, Al-Shehri MY, Malatani TS. Thyroid malignancy in multi-nodular goitre and in a solitary nodule. J.R. Coll. Surg. Edinb 1995; 40: 310-12.

[5] Memon W, Khanzada TW, Samad A, Kumar B. Incidence of thyroid carcinoma in multi-nodular goitres. Rawal Med J $2010 ; 35$.

[6] Gandolfi PP, Frisina A, Raffa M, Renda F, Rocchetti O, Ruggeri C, et al. The incidence of thyroid carcinoma in multi-nodular goitre: a retrospective analysis. Acta Bio Medica Ateneo Parmense 2004; 75:114-17.

[7] Pedamallu R, Pedamallu SB, Rama Rao K, Pedamallu CS. Incidence of occult carcinoma in multi-nodular goitre which was diagnosed on the basis of the histopathological findings. The Internet Journal of Surgery. 2008 Volume 17 Number 1.

[8] Cole WH. Incidence of carcinoma of the thyroid in nodular goitre. Semin Surg Oncol 1991; 7 (2): $61-63$.

[9] Sachmechi I, Miller E, Varatharajah R, et al. Thyroid carcinoma in the single cold nodules and in the cold nodules of multi-nodular goitres. Endocr Pract 2000; 6 (1): 110-12

[10] Koh KB, Chang KW. Carcinoma in multi-nodular goitre. Br J Surg 1992; 79 (3): 266-67.

[11] Najum ul Haq R, Ali Khan B, Ahmed Chaudhry I. Prevalence of malignancy in goitre-a review of 718 thyroidectomies. J Ayub Med Coll Abbottabad 2009; 21(4)

[12] Cerci C, Cerci SS, Eroglu E, Dede M, Kapucuoglu N, Yildiz M, et al. Thyroid cancer in toxic and non-toxic multi-nodular goitre. J Postgrad Med 2007; 53:157-60. 